\title{
A Study of Hydrogels
}

\section{Shailee V Tiwari* and Shruti V Dabhade}

Department of Pharmaceutical Chemistry, Durgamata Institution of Pharmacy, Parbhani, India

*Corresponding Author: Shailee V Tiwari, Department of Pharmaceutical Chemistry, Durgamata Institution of Pharmacy, Parbhani, India
Received: March 12, 2021

Published: May 24, 2021

(C) All rights are reserved by Shailee $\mathbf{V}$

Tiwari and Shruti V Dabhade.

\begin{abstract}
Hydrogels are hydrophilic, crosslinked polymeric structures that can swell in water and can maintain its structure by physical or chemical crosslinking of polymer chain. They are $90 \%$ water and permeable to drugs for delivery and encourage controlled delivery. Due to their physicochemical properties and preparation methods hydrogels are applied in large scale engineering and biomedical applications, regenerative medicine. In this article, different types of hydrogels are studied, followed by their preparation methods, classification, physicochemical properties, different characterizations, and their current trends and applications in various fields such as cell culture, clinical surgery, tissue engineering, biosensing, medication delivery and release are discussed.
\end{abstract}

Keywords: Hydrogels; Applications; Directed Drug Delivery; Drug Discharge

\section{Introduction}

The hydrogels are 3-dimensional, cross-connected organizations of water-soluble polymers. Further, they can be formed in structures like sections, microparticles, nanoparticles, coatings. Thus, hydrogels are normally utilized in clinical practice and trial medication for a wide scope of uses, for example, tissue designing and regenerative medication [1], diagnostics [2], cell immobilization [3], partition of biomolecules or cells [4] and boundary materials to direct natural bonds [5]. The properties of hydrogels have numerous benefits in their utilization in drug delivery applications. Their permeable construction can be changed by controlling the thickness of cross-joins in the gel network. Advantages of hydrogels for drug conveyance might be generally pharmacokinetic that a terminal detailing is made from which medicates gradually elute, keeping a high neighborhood grouping of medication in the encompassing tissues over an all-inclusive period. Hydrogels are additionally profoundly biocompatible, as considered in their fruitful use in the peritoneum and different locales in vivo. Biocom- patibility is upgraded by the high water level of hydrogels and the physiochemical likeness of hydrogels to local extracellular system, both compositionally (especially on account of sugar based hydrogels) and precisely. Biodegradability or disintegration might be planned into hydrogels by means of enzymatic, hydrolytic, or ecological (for example $\mathrm{pH}$, temperature, or electric field) pathways. Hydrogels are generally deformable and can adjust to the state of surface to which they are applied. The muco - or bioadhesive properties of certain hydrogels can be worthwhile in immobilizing them at site of utilization or in applying them on surfaces that are not flat. Significant are issues identifying with drug delivery properties. Especially if there should arise an occurrence of hydrophobic medications, amount and homogeneity of medication into hydrogels might be restricted. The high water fixation and huge pore sizes of most hydrogels may frequently bring about quick medication discharge, over a couple of hours to a couple of days. Simplicity of utilization can likewise be risky; as certain hydrogels are appropriately deformable to be injectable, many are not, requiring careful implan-

Citation: Shailee V Tiwari and Shruti V Dabhade. "A Study of Hydrogels". Acta Scientific Pharmaceutical Sciences 5.6 (2021): 86-97. 
tation. These issues fundamentally limit functional utilization of hydrogel-based drug delivery treatments in center. In this study, we center around issues in regards to utilization of hydrogels for drug delivery: encouraging the in vivo use of medication eluting hydrogels, expanding their span of medication delivery, and widening the scope of medication [6].

Types of hydrogels [7-15]

\section{Conductive hydrogels}

Conductive hydrogel is a new viable material which has the comparative remarkable properties to traditional hydrogels and an extra advantage in electrical conductivity [9-11]. It was first proposed by Gilmore., et al. [12]. Depending upon various added substances, they are divided into two classes: (i) the conductive hydrogels-based conducting polymers and (ii) the conductive hydrogels-based metallic nanoparticles. Mostly the conductive hydrogels is defined as a mixture network made by cross-linked soft hydrogels and conducting polymers [13]. In spite of the fact that conducting polymer is not metallic, it has unprecedented electrical conductivity that can change final hydrogels in primary and electrical properties to a significant degree. For example polyacetylene, poly (phenylene vinylene), polythiophene $[14,15]$.

\section{Injectable hydrogels}

Injectable hydrogels are described by intrinsic fluidity and can be applied by an infusion technique. Injectable hydrogels show solgel stage transition. This not just grows the extent of its application in the biomedical field yet in addition improves comfort satisfaction of patients. Injectable hydrogels can be isolated into two classifications: light irradiation hydrogels and self assembling hydrogels. Light irradiation hydrogels include formation of irreversible covalent bonds through utilization of visible or UV, while selfassembling hydrogels are shaped immediately or after directional initiation. For example, poly (vinyl alcohol), poly (ethylene glycol).

\section{Double network hydrogels}

Double network hydrogels contain two interpenetrating polymer network; one of which is a cross-linked rigid polymer network, while the other is a gently cross-linked flexible polymer network. While this construction is associated physically, no substance cross- linking happens between these two commonly free cross-linked network. Hydrogels show a specific viscos flow because of loose cross-linking of the subsequent network, which can adequately absorb fracture energy through the network deformation as well as the slippage of physical entanglements along the polymer chain. This prevents breaks from proliferating across the structure, so they have great mechanical strength. Notwithstanding more mechanical strength, hydrogels have a few different benefits over single network structure, for example poly(N,N'-diethylacrylamide).

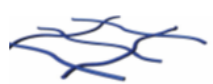

(a)

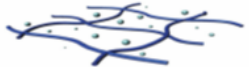

(b)

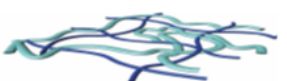

(c)
Figure 1: Schematic outline of the creation cycle of interpenetrating polymer network hydrogels.

\section{Responsive hydrogels}

Hydrogels with responsive performance have a wide scope of applications in natural tissue designing, including drug delivery and controlled release, artificial muscles, sensors, and protein catalysis. At the point when ecological changes (e.g. outside temperature, $\mathrm{pH}$, light, electric field, and saltiness) happen, hydrogels can shrink or swell on a case by case basis because of the presentation of hydrogen bonds, ions, complexation, electrostatic and other noncovalent interactions. These responsive "smart" or clever hydrogels were first evolved by Katchalsky in 1949, by copolymerizing methacrylic corrosive with a low level of divinyl benzene, delivering a gel capable for retaining hundreds of time its own weight of water at higher $\mathrm{pH}$ value, then shrinking with diminishing $\mathrm{pH}$.

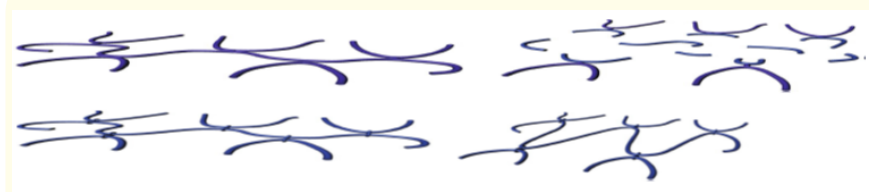

Figure 2: General science hydrogel and sliding hydrogel. 


\section{Nanocomposite hydrogels}

The nanoscale scattering of various materials in polymeric matrix from nanocomposite hydrogels, with molecule sizes going from 1 to $1000 \mathrm{~nm}$, and molecular chain structure comprises of branched polymers and cross-linked network polymers [9]. This uniform dispersion of inorganic component can enormously improve strength of hydrogels [8]. Nanocomposite hydrogels,that are also called hybrid hydrogels, which are made by physical or chemical covalent cross-linking of polymer network with raw material particles, including c-based nanoparticles, polymeric nanoparticles, inorganic, metal/metal oxides $[8,9]$.

\section{Sliding hydrogels}

Sliding hydrogels has topologically interlocked noncovalent cross-links that slide along threaded polymer spine A noval sliding hydrogel, pseudopolyrotaxanes of monothiolated beta-cyclodextrin strung on poly(allyl glycidyl ether)- block-poly(ethylene glycol)- block-poly(allyl glycidyl ether), was recently arranged in water by sonication and therefore photocrosslinked by ultraviolet light, bringing about a sliding hydrogel with elasticity comparableto different hydrogels, enhanced stretchability, furthermore, tunable degradability under acidic conditions [7]. The advantage of these hydrogels lies in their more stable mechanical properties, as other hydrogels show more volality.

\section{Other novel hydrogels}

Notwithstanding the five biomedical hydrogels referred to above, research has prompted the turn of events of various other novel hydrogels, including DNA-enabled hydrogels, and magnetic hyaluronate hydrogels. DNA-enabled hydrogels have been produced through reaction of dibenzo cyclooctyne-functionalized multiarm poly (ethylene glycol) with azide-functionalized singleabandoned DNA in aqueous solution by without copper click science.

Preparation methods of hydrogels [16-19]

Hydrogels can be prepared using different method of crosslinking are as per the following.

\section{Crosslinking of polymers method}

In this technique artificially crosslinked gels are made by total polymerization of low molecular weight monomers, or extended homopolymers, or copolymers with crosslinking factor. This is generally done for biomedical applications. These are non reversible reaction after synthesis. They are also known as thermosetting polymers. Example bakelite, phenol formaldehyde polymer, resins, urea formaldehyde polymer.

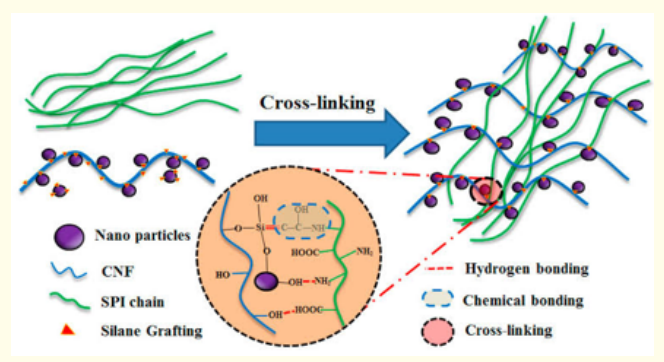

Figure 3: (a). Hydrogel preparation by Crosslinking polymer method.

\section{Copolymerization/crosslinking responses method}

Mixture of different monomeric samples are polymerized and form copolymer, this process is called as copolymerization. Copolymerization responses are utilized to produce polymer gels, numerous hydrogels are prepared. Example., Nylon 66 is copolymer of hexamethylenediamine and adipic acid.

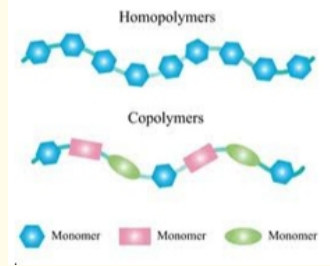

Figure 3: (b). Hydrogel preparation by copolymerization method.

\section{Crosslinking by high energy radiation method}

In this method gamma ray and electron ray radiation can be used to polymerize unsaturated substances. $\mathrm{H}_{2} \mathrm{O}$-soluble polymers derivatized with vinyl moiety can be converted into hydrogels. Example, polypyrrol, polyvinylpyrrolidone.

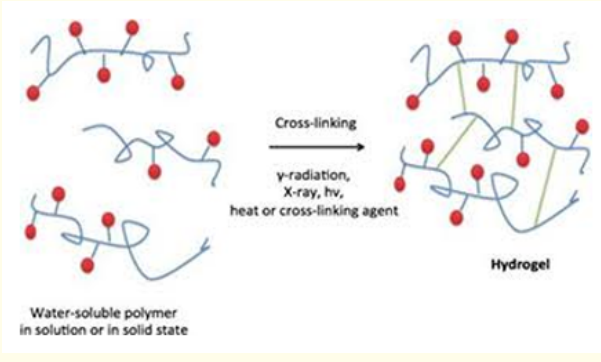

Figure 3: (c). Hydrogel preparation by crosslinking by high energy radiation method. 


\section{Crosslinking using enzymes method}

Another technique was discovered utilizing a compound to produce polyethyleneglycol based hydrogels. A tetrahydroxy polyethylene glycol was functionalized with expansion of glutaminyl gatherings and organization were shaped by expansion of transglutaminase into arrangement of polyethyleneglycol.

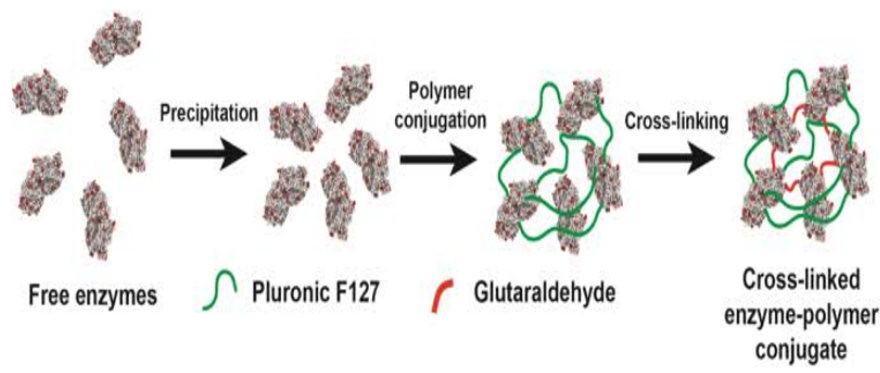

Figure 3: (d). Hydrogel preparation by crosslinking using enzyme method.

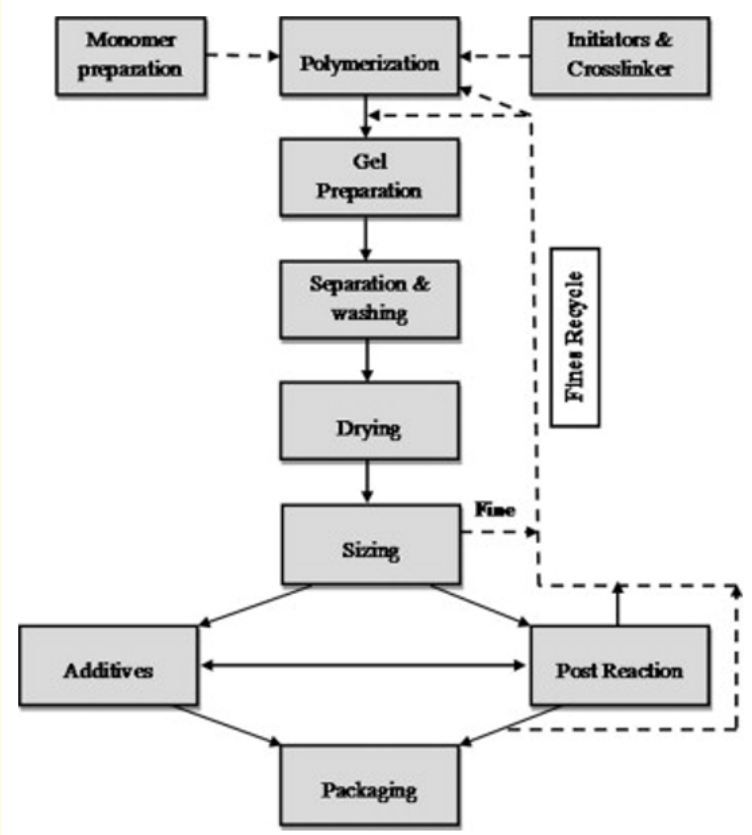

Figure 3: (e). Hydrogel preparation block diagram (solution polymerization procedure).

Classification of hydrogels [20]

On basis of technique for preparation, hydrogels are categorized into

- Homopolymer hydrogels: They are derived from single species of monomer and may have crosslinked skeleton depend- ing on nature of polymer and polymerization technique they are used in drug delivery system and contact lenses. Example, Polyethyleneglycol is widely used in drug delivery system due to high responsive towards external stimuli.

- Co-polymer hydrogels: They are made up of two types of monomers in which one is hydrophilic in nature. The ring opening copolymerization of caprolactone (nylon 6) technique is used here. Example, poly(ethylene glycol)poly(caprolactone)-poly(ethylene glycol) triblock copolymeric hydrogel are used in drug delivery system.

- Multi polymer hydrogels: They are composed of two independent crossed linked synthetic or natural polymer. These are done by immersing pre-polymerised hydrogel in solution of monomer and polymerization initiator. Example, inter penetrating network polymer can overcome thermodynamic incompatibility.

On basis of instrument controlling the medication discharge they are categorized into

- Diffusion controlled delivery: Diffusion is common mechanism. There are two types reservoir devices and matrices devices. Reservoir devices are capsules, cylinders, slabs, spheres. Outer membrane of device is rate limiting step for drug release. In matrices devices drug diffuses from macro molecular mesh or water filled pores.

- $\quad$ Swelling controlled delivery: Here drug is dispersed within glassy polymer. When it comes in contact with biological fluid, polymer swells. For relaxation of macro molecular chain glass transition temperature of polymer is lowered.

- Chemically controlled delivery: There are two types erodible drug delivery system and pendent chain system. Drug release occurs due to degradation or dissolution of hydrogel in erodible drug delivery system. And in pendent system polymer back bone is affixed by drug through degradable linkage. Degrade drug is released by the linkage.

- Environment responsive: There are several environmental stimuli that discharge drug for example ph, temperature, physical stimuli such as light, magnetic field, electric current, ultra sound and chemical stimuli like certain ionic species and biological compounds. 
On basis of ionic charges hydrogels can be categorized into

- $\quad$ Neutral hydrogels (non ionic): Neutral hydrogels are those originating from acrylamide, n-vinyl pyrrolidone, hydrophobic acrylic acid. Example, hydroxylalkyl methacrylates.

- Anionic hydrogels (ionic): Anionic hydrogels are that originating from arylic acid and crotonic acid. Example, hyaluronic acid, pectin, carrageenan, chondroitin sulfate, dextran sulfate.

- Cationic hydrogels (ionic): Cationic hydrogels are those having origins in amino ethyl methacrylate. Example, vinylpridine, chitosan, polylysine.

- Ampholytic hydrogels (acidic and basic group both): Ampholytic hydrogels contains both acid group and basic group in their structure. They are prepared by [(methylacrylamido) propyl]trimethyl ammonium chloride and sodium sulfonate copolymerizing with acrylamide. Example, sodium carboxymethyl cellulose.

On basis of construction hydrogels can be categorized into

- Amorphous hydrogels: Amorphous hydrogels are glycerine-water based formulations used for wound healing. They provide moisture to dry wounds. They are used generally for necrosis wound, minor burns and radiation tissue damage. Example, pectin, glycerine, carboxymethylcellulose, propylene glycol.

- Semi-translucent hydrogels: Semi-translucent hydrogels are formed by penetrating linear polymer into other crosslinked network without other chemical bond between them. They are able to maintain a fast kinetic response. Example, polyethyleneglycol.

- Hydrogen bonded-hydrogels: Hydrogen bonded hydrogels are transparent hydrogels. Hydrogel ability to absorb large amount of water is enhanced by hydrogen bonding. Example, xanthan, polymer-polymer complex, matured acacia gum.

On basis of sources hydrogels can be categorized into

- Natural hydrogels: Natural hydrogels are biodegradable with low toxicity byproduct. They remain most physiological hydrogel as they are extracellular matrix component in vivo. Example, dextran, chitosan, collagen, dextran sulfate, fibrine, matrigel, hyaluronic acid, cellulose, sodium alginate.
- Synthetic hydrogels: Synthetic hydrogels have strong covalent bond within matrix improving mechanical strength. They are hydrophobic in nature, with low immunogenicity, low biodegradability and can be designed to meet specific needs, can be mass produced and precise control. Example, polyvinyl alcohol, polyethylene oxide, poly propylene fumarate.

- Hybrid hydrogels: Hybrid hydrogels consist of functionally, morphologically, chemically different polymers which has biologically active peptides, protein or micro/nano structures interconnected that are physically or chemically. Examples, collagen-acrylate, poly(polyethyleneglycol-co-peptides).

Physicochemical properties of hydrogels [20]

The physicochemical properties of polymers help to decide the usefulness of the hydrogel. These physicochemical properties depend on the $\mathrm{pH}$ of hydrogels, chemical nature of the monomer, density, degree of polyrization, crystallinity of material, the technique used for polymer preparation, molecular weight, molar volume, environmental stimuli, and macromolecular structure. The design of the polymer, depends on the strength of covalent bonds, the rigid nature, and intermolecular force strength inside the polymer chain. They maintain the physical structure in drug delivery. They are non toxic, degradable, non immunogenic, has stiffness, elasticity, shearthinning property, sol-gel transition stimulus and are conventional.

\section{Characterization of hydrogels}

Hydrogels are described for their morphology, growing property and flexibility. Morphology is characteristic of their permeable construction. Growing decides the delivery system of the medication from the swollen polymeric mass while flexibility influences the mechanical strength of the organization and decides the steadiness of these medication transporters.

Morphology

Hydrogels are described for morphology which is dissected by stereomicroscope. Likewise the surface of these biomaterials is dissected by scanning electron microscopy to guarantee that hydrogels, particularly of starch, hold their granular constructions. They are indicative of their porous structure. The surface morphology of hydrogels is studied by a digital instrument called as multimode atomic force microscope [21].

\section{X-ray diffraction}

It is used to know whether the polymers hold their translucent design or they get disfigured during the handling compression measure $[21,22]$. 
In-vitro discharge concentrate for drugs

Since hydrogels are the swollen polymeric organizations, inside of which is involved by drug particles, discharge study is done to know the way of delivery over a time of utilization [21,22].

\section{Fourier transform infrared spectroscopy}

Any adjustment in morphology of hydrogels changes infrared retention spectra because of extending and $\mathrm{O}-\mathrm{H}$ vibration. Development of helix which is characteristic of cross connecting is clear by appearance of group close 1648 1/cm [22].

\section{Swelling factor}

The hydrogels are permitted to soak in aq. medium or mode of explicit pH to know the swell ability of these polymeric organizations. These polymers show increment in measurements identified with swelling $[23,24]$.

\section{Rheology}

Hydrogels are assessed for consistency under steady temperature of normally $4^{\circ} \mathrm{C}$ by utilizing Cone Plate type viscometer [25].

\section{Application and current trends of hydrogels}

\section{Cell culture}

Research on cell biomaterials in the most recent decade has moved in the direction of 3-D approaches [26]. Hydrogels like VitroGel and GelMA have been demonstrated to be non-cytotoxic from multiple points of view and are in this manner broadly utilized in 3D cell culture [27-30]. Figure 4 shows different hydrogel has a reversible fluorescent staining [31]. Since the hydrogel has great biocompatibility, it is utilized as a three-dimensional culture medium for cells, and the fluorescence sign of the network hydrogel can be controlled into "on" and "off" states during the perception cycle, which is includes multicolor fluorescent stained cells.

\section{Clinical surgery}

Uncontrolled bleeding outcomes in over $30 \%$ of traumatic deaths, the greater part of which happen before emergency care arrive. For deep injuries specifically, it is hard to quit bleeding by pressure or a tourniquet because of the unpredictable shape of the injury, and as of now, the hemostasis regularly utilized in a medical procedure does not show satisfactory effect on deep injuries. Zhao utilized chitosan derivative and carbon nanotube materials to get ready injectable shape memory nanocomposite permeable colloidal hemostatic materials, appeared in figure 5 [32]. As the principle skeleton of crystal gel, chitosan derivative give great hemostatic properties and increase injury healing and blood-triggered shape

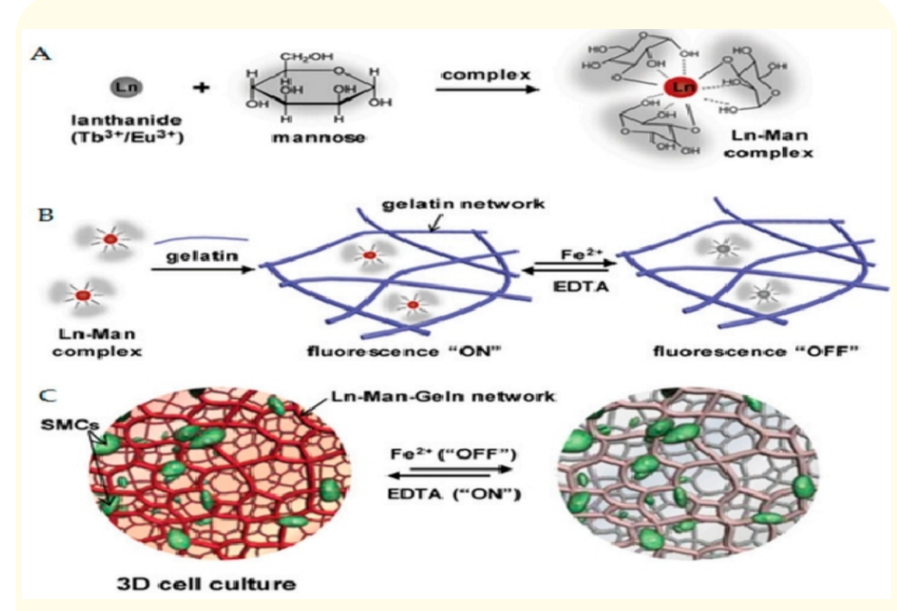

Figure 4: A schematic graph of the arrangement of a reversibly responsive fluorochromic hydrogel in view of a lanthanide-mannose complex. (A) Scheme of the development of lanthanide-mannose complex(B)Scheme of the arrangement of lanthanide-mannosegelatin hydrogel by presenting Ln-Man into the gelatin organization (C) 3D cell culture network.

memory recovery. As a nanofiller, the carbon nanotube material can improve the mechanical properties of the gel, and the carbon nanomaterial can additionally improve the procoagulant effect of composite gel. The composite cell's platelet and platelet adsorption and activation capacities permit it to show good hemostatic properties in many deep injuries. The shape memory composite gelatin hemostatic dressing has low raw material expense and has good application potential in deep injury hemostasis.

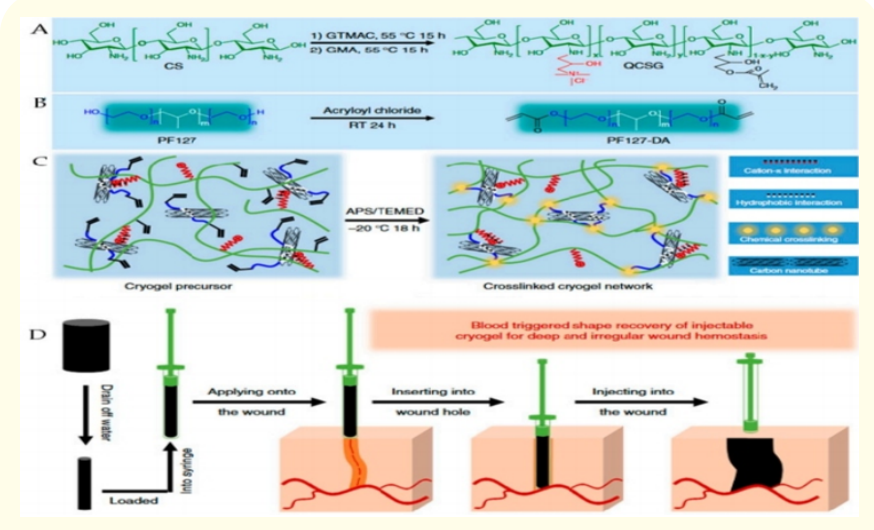

Figure 5: Schematic portrayal of glycidyl methacrylate functionalized quaternized chitosan/carbon nanotube cryogel union and application. (A) Synthesis of copolymer. (B) Synthesis of copolymer. (C) Preparation of cryogel. (D) Schematic portrayal of hemostatic use of injectable shape memory cryogel hemostatics. 


\section{Tissue engineering}

Defect of irregular bone or deformities like ligament, ligaments, and tendons, are a clinical issue in the treatment of bone defect. Autologous bone grafting is as yet the best option for bone fix, yet limited source of autologous bone and the tendency of injury development in the donor site limit its application. Hence, tissue designing scaffolds that encourage insignificantly obtrusive medical procedure and versatile unpredictable bone deformities and enhance bone recovery are relied upon to be ideal bone repair materials.

\section{Biosensing}

Utilized for vision correction as a compact and available gadget, with great biocompatibility with many years of clinical use have now grabbed a lot of eye in drug delivery and tear analyte detection field [33]. Chen built up progression of glucose hydrogel-based colloidal photonic crystal materials. A hydrogel was crosslinked from the polyethylene glycol system, which could give antifouling layer for entry of glucose and modify with boronobenzaldehyde (Figure $6 \mathrm{~A}$ ), improving affectability for glucose A model of such lens is appeared in figure 6B. In figure 6C frequency shifts at low glucose level. Boronic produce protons by extracting a hydroxide unit from $\mathrm{H}_{2} \mathrm{O}$ [33]. Then again 2D colloidal photonic crystal glucose sensors, are monolayered designs of as appeared in figure $6 \mathrm{~d}$. Phenylboronic molecular response with glucose has (Figure 6E) (1) boronic bind glucose in neutral 3-side structure; (2) hydroxylation of phenylboronic in basic medium, $\mathrm{OH}$ attacks $\mathrm{B}$ results conformity progress of phenylboronicintetrahedral (3) formed tetrahedral anion of phenylboronic mix with starch [34].

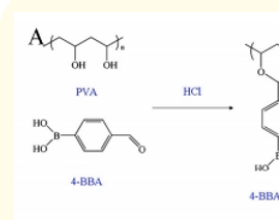

D

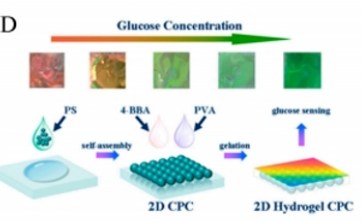

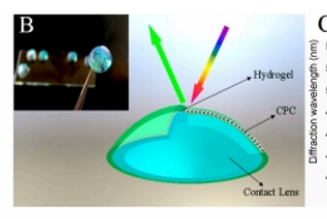

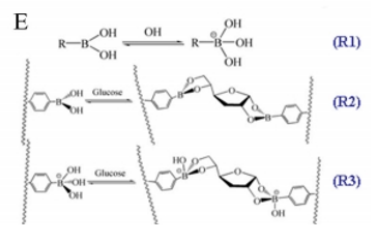

Figure 6: (A) Diagram of a hydrogel colloidal photonic crystal detecting focal point. (B) The reaction at low glucose fixation (C) Reaction between polyvinyl alcohol and 4-boronobenzaldehyde.

(D) Illustration of the development of the polyvinyl alcohol hydrogel colloidal photonic crystal, (E) Scheme of harmony between boronic corrosive and glucose in weaken arrangement: boric corrosive and borate particles can be shaped (R1), and boronic corrosive can reversibly tie glucose both in its impartial three-sided structure (R2) , its charged tetrahedral structure (R3).
Medication delivery and release

Nanodrugs have incredible potential and application possibilities in clinical therapy of cancer. Nanomedicine is made out of transporters and chemotherapeutic medications. Medications play a therapeutic job, and transporters play embedding and transporting job. Polymer micelles have pulled in much consideration in view of their great biocompatibility, simple design change, also, "core shell" structure. Li developed cisplatin-crosslinked polymer prodrug micelle nanodrugs with a double stabilization by coordination complexation and synergistic stabilization of polymer prodrugs. Figure 7 shows the design and arrangement of polyethylene glycol-polyglutamic acid with great biocompatibility as the fundamental polymer chain and the camptothecin antecedent with a disulfide bond as the linker. The hydrophobic properties of camptothecin were utilized to build the polymer precursor micelles. At last, the polymer precursor micelles with twofold stability were built by complexing carboxyl gatherings with cisplatin [35].

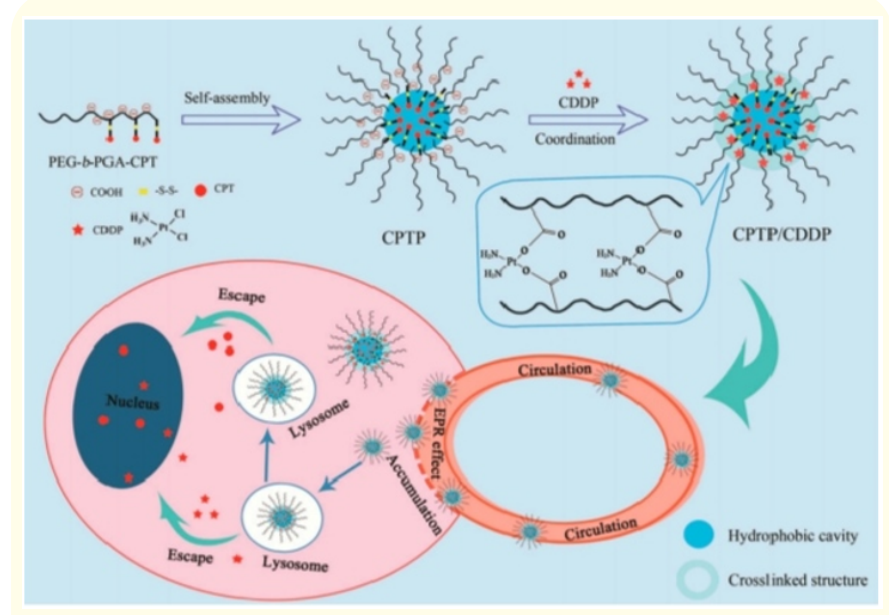

Figure 7: Schematic representation of double stable nanomedicines built by cisplatin-crosslinked camptothecin prodrug micelles for better drug delivery.

\section{Supramolecular hydrogels}

The supramolecular hydrogel system is made out of intermolecular collaborations that are noncovalent also, has at least two atomic substances held together Advancement has been made with supramolecular drug loading and gelation at the same time in a aqueous environment hydrogels utilizing self-assembled incorporation complex among cyclodextrins that give maintained and controlled arrival of macromolecular medication [36].

Common cyclic oligosaccharides made out of six, seven or eight glucose units connected by 1,4-linkages are called cyclodextrins and 
are appropriate for use in supramolecular system. A new report included improvement of a glycoconjugate arranged by amidation of homopoly- levo-guluronic corrosive square acquired from dextran. antarctica sodium alginate with mono-6-amino- $\beta$. The study was pointed toward treating Chagas illness brought about by Trypanosoma cruzi. Lipophilic nonhydroxylated coumarins were loaded into the hydrophobic center of the $\beta$-cyclodextrin to deliver them with trypanocidal action. Cooperation between the carboxylate gatherings of unconjugated. Lglucuronate buildups with calcium particles was utilized to deliver supramolecular hydrogels of glycoconjugate of homopoly-levo-guluronic block division with 6- $\mathrm{NH}_{2}-\beta$. As the T. cruzi parasites have just a single mitochondrion, it is an ideal objective for medications to control its energy cycle also, apoptosis. Mitochondrial layer potential investigations uncovered that the cyclodextrin complex with the medications delivered critical oxidative pressure to obliterate the parasites. The medication in the complex had expanded solvency, showed improved bio-accessibility, controlled medication discharge and improved trypanocidal movement in contrast with the relating free amidocoumarins [37]. Cyclodextrin-functionalized polyhydrazines were utilized to plan hydrogels in-situ by means of hydrazine bond arrangement with aldehyde bunches on dextran aldehyde. No harmfulness was seen in vitro with these hydrogels and they could oblige nicardipine as hydrophobic medication into the cyclodextrin cavities. Consistent arrival of nicardipine more than 6 days was seen with the hydrogel planning having higher hydrazine linkages. Accordingly, a gel equipped for hydrophobic medication discharge in an in-situ shaped gadget over expanded periods was created [38]. Bleeding control and wound healing by bio-adhesive hydrogels find many biomedical uses. In situ forming hydrogels are utilized to recuperate harmed tissues dependent on their capacity to collect and produce a fibrin connect that grant fibroblast movement and collagen discharge for healing tissue injury. $\beta$-Cyclodextrins are a non-poisonous adjuvant for drug and mucoadhesive applications. Somewhat oxidized $\beta$-cyclodextrin was utilized in a new report to misuse aldehyde gatherings on a hydrogel grid for ideal response with amines in the tissue to bring about an imine bond (Schiff's base response) to adhere to the skin and to give improved cyclodextrin solvency to improve loading proficiency. Mixing gelatin with the $\beta$-cyclodextrin incompletely oxidized with oxidation in presence of $\mathrm{H}_{2} \mathrm{O}_{2}$ /horseradish peroxidase, brought about fast development of gelatin- $\beta$-cyclodextrin hydrogels (Figure 8). Hydrophobic medications for example, dexamethasone could be delivered with 2.7 folds higher adequacy when delivered in presence of the cyclodextrin comparative with the gelatin-just hydrogels [39].

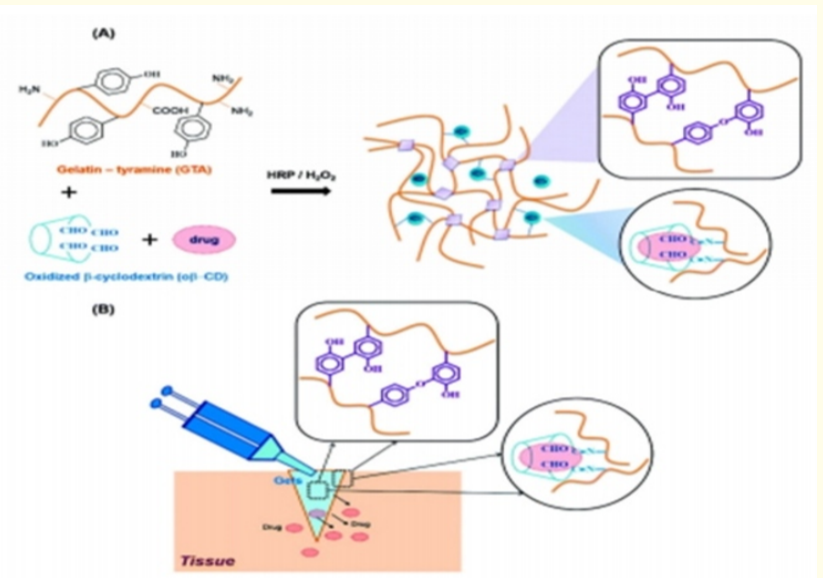

Figure 8: (A). Graphical portrayal of strategies for cross-connecting to get gelatin- $\beta$-cyclodextrin hydrogels to stack hydrophobic medications. (B). Schematic portrayal of glue hydrogels in situ shaped by consolidating to catalysis and the Schiff base response with restorative delivery.

\section{DNA-Hydrogels}

Hybrid bionanomaterials could be created utilizing DNA as the structure block. 2 or 3-dimensional constructions are shaped from DNA particles. High dissolvability, bio-similarity, adaptability and responsiveness are vital highlights of such hydrogels. Aside from these highlights they can likewise be labeled with appropriate fluorescent particles for tracking organic examinations in vitro [40]. A fascinating use of hydrogels has been made with the improvement of multi-useful quantum dot DNA hydrogels. DNA hydrogels are made out of correlative strands of DNA hybridized to shape a crosslinked network that swells in aqueous environment. The singlestep assembling zinc sulfide Doxorubicin DNA hydrogels showed expanded tumor aggregation in vitro, high bio-similarity, was triple more solid than free Doxorubicin and filled in as a great apparatus for in vivo bio-imaging in observing tumor development after some time. Aptamers, for example, siRNA were utilized to target specific cell types to deliver drug specifically and adjust protein expression in different cell types (Figure 9) [41].

A model is the development of supramolecular hydrogel with tunable mechanical properties and multi-shape memory impacts. These hydrogels utilize agar that is actually cross-connected to shape the hydrogel organization and a supramolecular network that is cross-connected by appropriate chemical bonds that fix 

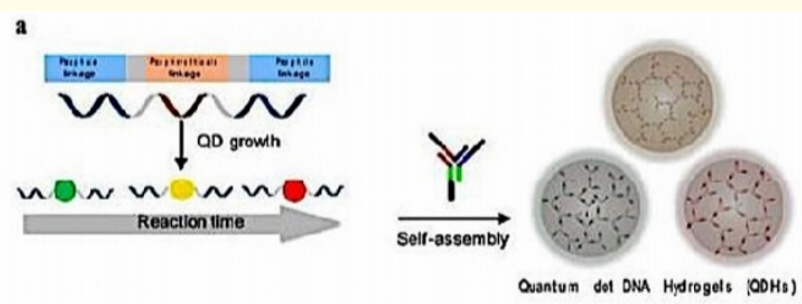

b

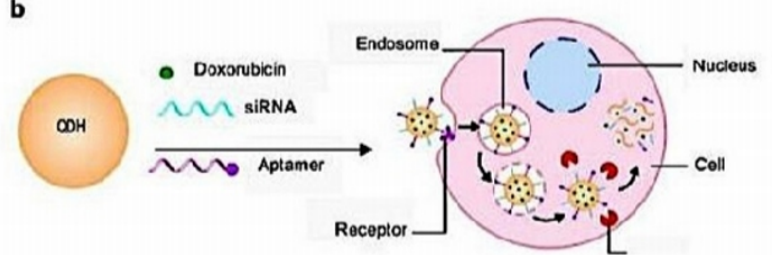

Figure 9: (a). Schematic perspective on the synthesis process of the DNA functionalized doxorubicin followed by the development of the doxorubicin hydrogel through hybridization with DNA. (b). Schematic perspective on the modulation process on the hydrogel for specific targeting of cell utilizing aptamer for drug delivery.

shapes temporarily and produces a multi-shape memory impact made conceivable by reversible cooperation's (Figure 10). Supramolecular hydrogel has tremendous biocompatibility furthermore; bio-degradability attributes and depends on non-covalent interactions to drive the self-get together of small atoms in water. The shaped designs have supramolecular structures [41,42].

\section{Bio-inspired hydrogels}

A new type of hydrogels utilized for drug delivery applications are the bio-inspired hydrogels. These 3D materials reiterate the biological micro-environment applicable to the illness condition, support study on how the targeted drug delivery process could be improved, how the treatment acted in vivo, how the infection advanced, etc. These are especially valuable in cancer treatment as the illness is especially unpredictable and regularly connected with complex cell and physiological changes that require progressive observing. Designing such microenvironments would accordingly be a valuable way to deal with advance examination and study the illness condition and helpful interaction better. The stiffness of the 3D model utilized to study liver cancer is a basic characteristic to control atomic diffusivity and threat. The flexible moduli of the collagen gels were expanded by stiffening interconnected collagen fiber with varied amount of poly(ethylene glycol) di(succinic corrosive N-hydroxysuccinimidyl ester). The softer gels delivered threatening disease spheroids while the stiffer ones showed stifled

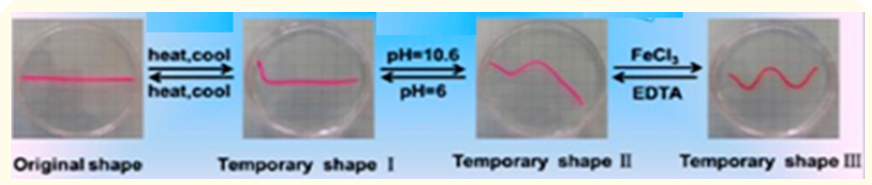

Figure 10: Schematic portrayal of multi-shape memory effect.

danger. The model gave better understanding and guideline of the developing practices of cancer cell growth [43].

Bio-inspired have been grown as with improved medication delivery properties fit to perfectly coordinate the eye condition in the infected state, particularly of the anterior eye fragment. Sustained medication levels in tear liquid for a prolong time according to eye drops was noticed. The medication timolol was delivered in reaction to lysozyme in the medium. Catalyst cleavable polymers in hydrogels empowered this. Ergosterol-liposome united silicone materials loaded with nystatin taken after a parasitic contamination and set off nystatin discharge through a serious instrument. In the absence of the ergosterol (significant sterol in the cell layers of growths) in the medium, the medication discharge was entirely negligible [44-47].

\section{Multi-functional and stimuli-responsive hydrogels}

Hydrogels that were multi-functional and transporters of anticancer drugs are example of these delivery vehicles and their amiability to substance changes to upgrade their remedial impacts. Magnetite nanoparticles that upheld increased intracellular takeup by cells additionally had folate ligand on them to empower targeted delivery. In addition, the hydrogel polymers were thermally responsive and doxorubicin loaded. Those altered hydrogels offered benefits like increased cell take-up and apoptotic movement in vitro [48]. Another improvements responsive hydrogel grew as of utilized biocompatible thermally responsive polymers that encouraged crack of cancer cells. The examination was directed in vitro with an outer wellspring of warmth and showed effective cell burst. Those hydrogel particles had (Arginine, Glycine, Aspartate) peptides joined to their surface that could tie to cells (Figure 11).

Since in vivo conditions in tumors typically produce a higher temperature than the surrounding regions, and furthermore that it has been demonstrated that the actual power created from the extension of the cells when they were warmed was adequate to execute them, that kind of thermally responsive hydrogels end up being profoundly beneficial alternatives for accomplishing targeted 

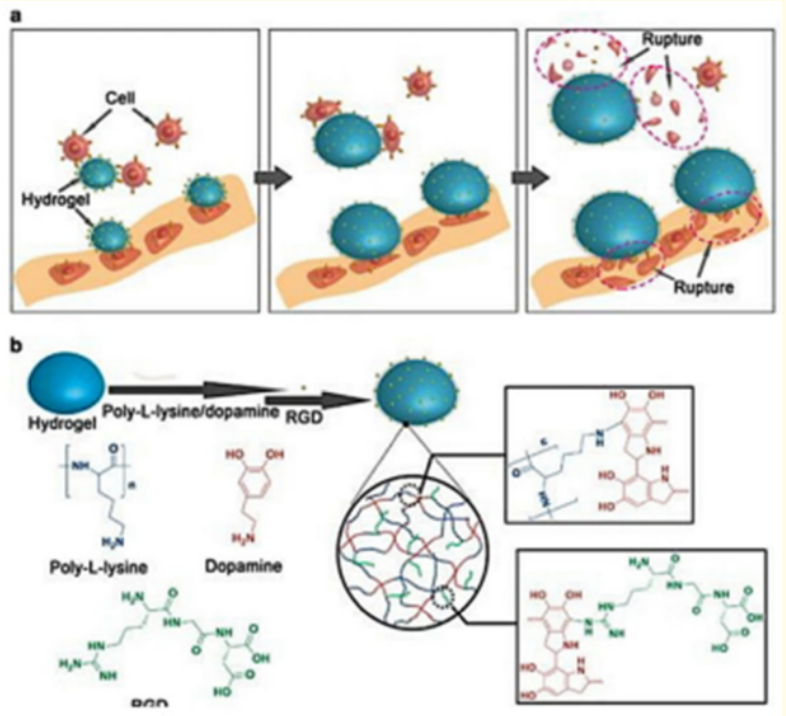

Figure 11: (a). Schematic representation of the disease cell connection on upgrades responsive hydrogel (b). Schematic portrayal of the surface adjustment of hydrogel.

on cell killing. Hydrogen peroxide trigger-mediated release of medications could be accomplished utilizing hydrogels. This has been shown with the utilization of $\mathrm{ABC}$ type triblock copolymer poly [(propylenesulfide)- b-(N,N-dimethylacrylamide)- b-(N-isopropylacrylamide)] [49]. The gelation to form the cross-linked hydrogel occurred over $37^{\circ} \mathrm{C}$ and the encompassing $25^{\circ} \mathrm{C}$ temperature was adequate to encapsulate the hydrophobic medication through the formation of micelles. Consequently, the temperature-responsive polymer could cross-connection to be a stable hydrogel carrying the hydrophobic medication dependent on the temperature change and furthermore showed a release of the red shaded color dependent on the $\mathrm{H}_{2} \mathrm{O}_{2}$ release in the environment (Figure 12) [50].

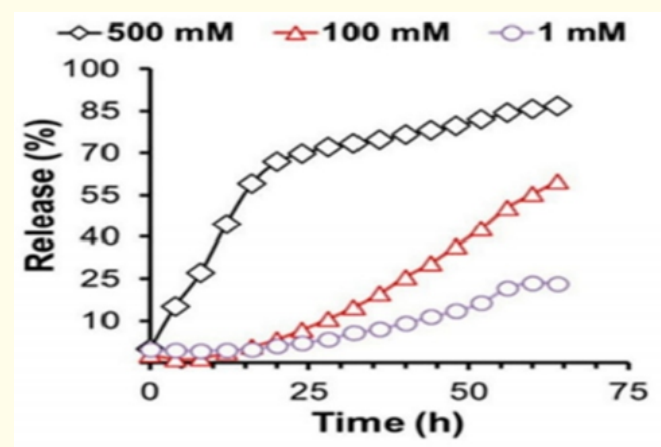

Figure 12: Medication discharge subject to Reactive Oxygen Species fixation.

\section{Conclusion}

The hydrogels depending on network are designed to meet different applications. When these hydrogels are in contact of aqueous solution are able to swell. The article demonstrates about the types and classification of hydrogels on different basis, their different methods of preparations, physical and chemical characteristics, and numerous applications. The study shows that hydrogels have unique properties that many future applications as next generation biomaterials. Therefore hydrogels are known as smart or intelligent biomaterials. There are various current trends of hydrogels. Some are discussed in this article.

\section{Acknowledgement}

I am very much thankful to the Principal Dr Sameer Shaikh, Dr Shailee Tiwari and all the teaching and non teaching staff of Durgamata Institute Of Pharmacy for support in completion of this article.

\section{Bibliography}

1. Lee KY and Mooney DJ. "Hydrogels for Tissue Engineering". Chemical Review 101.7 (2001): 1869-1880.

2. Van der Linden HJ., et al. "Stimulus-sensitive hydrogels and their applications in chemical (micro)analysis". Analyst 128 (2003): 325-331.

3. Jen AC., et al. "Hydrogels for cell immobilization". Biotechnology and Bioengineering 50.4 (1996): 357-364.

4. Wang K., et al. "Hydrogels as separation agents". Advances in Polymer Science 2 (1993): 67-79.

5. Bennett SL., et al. Journal of Cardiac Surgery 18.6 (2003): 494499.

6. Sutton C. The Obstetrician and Gynaecologist 7 (2005): 168176.

7. Murakami T., et al. "One-pot "click" fabrication of slide-ring gels”. Macromolecules 48.21 (2015): 7774-77781.

8. Merino S., et al. "Nanocomposite hydrogels: 3D polymernanoparticle synergies for on-demand drug delivery". ACS Nano 5 (2015): 4686-4697.

9. Gaharwar AK., et al. "Nanocomposite hydrogels for biomedical applications". Biotechnology and Bioengineering 111.3 (2014): 441-453. 
10. Balint R., et al. "Conductive polymers: towards a smart biomaterial for tissue engineering”. Acta Biomaterialia 10.6 (2014): 2341-2353.

11. Kloxin AM., et al. "Mechanical properties of cellularly responsive hydrogels and their experimental determination". Advanced Materials 22.31 (2010): 3484-3494.

12. Gilmore K., et al. "Preparation of hydrogel/conducting polymercomposites". Polymer Gels and Networks 2.2 (1994): 135143.

13. Sharma K., et al. "Conducting polymer hydrogels and their applications". Conducting Polymer Hybrids Springer, Cham (2017): 193-221.

14. Tang Q., et al. "Two-steps synthesis of a poly (acrylate-aniline) conducting hydrogel with an interpenetrated networks structure". Carbohydrate Polymers 67.3 (2007): 332-336.

15. Tang Q., et al. "A multifunctional hydrogel with high conductivity, pH-responsive, thermo-responsive and release properties from polyacrylate/polyaniline hybrid". Carbohydrate Polymers 73.2 (2008): 315-321.

16. Peppas NA. "Hydrogels in Medicine and Pharmacy". Fundamentals, CRC Press, BocaRaton, FL. 1986,1,180.

17. Malcolm B., et al. "Swelling properties of copolymeric hydrogels prepared by gammairradiation". (1986): 457-475.

18. Sperinde JJ and LG Griffith. "Control and Prediction of Gelation Kinetics in Enzymatically Cross-Linked Poly (ethylene glycol) Hydrogels". Macromolecules 33 (2000): 5476-5480.

19. Peppas NA. "Crystallization of polyvinyl alcohol-water film by slows dehydration". European Polymer Journal 12.8 (1976): 495-498.

20. Burkert S., et al. "Crosslinking of poly (N-vinyl pyrrolidone) films by electron beam irradiation". Radiation Physics and Chemistry 76 (2007): 1324-1328.

21. Szepes A., et al. "Characterization and drug delivery behaviour of starch basedhydrogels prepared via isostatic ultrahigh pressure". Carbohydrate Polymers 72 (2008): 571-575.

22. Yu H and Xiao C. "Synthesis and properties of novel hydrogels from oxidized Konjac glucomannan crosslinked gelation for in-vitro drug delivery". Carbohydrate Polymers 72 (2008): 479-489.
23. Yin Y., et al. "Study of the swelling dynamics with overshooting effect of hydrogels based on sodium alginate-g-acrylic acid". Carbohydrate Polymers 71 (2008): 682-689.

24. Kim SW., et al. "Hydrogels: Swelling, drug loading and release". Pharmaceutical Research 9.3 (1992): 283-290.

25. Schuetz YB., et al. "A novel thermo responsive hydrogel of chitosan". European Journal of Pharmaceutics and Biopharmaceutics 68 (2008): 19-25.

26. Pampaloni F., et al. "The third dimension bridges the gap between cell culture and live tissue". Nature Reviews Molecular Cell Biology 8 (2007): 839-845.

27. Yue K., et al. "Synthesis, properties, and biomedical applications of gelatinmethacryloyl (GelMA) hydrogels". Biomaterials 73 (2015): 254-271.

28. Nichol JW., et al. "Cell-laden micro engineered gelatin methacrylate hydrogels". Biomaterials 31 (2010): 5536-5544.

29. Loessner D., et al. "Functionalization, preparation and use of cell-laden gelatin methacryloyl-based hydrogels as modular tissue culture platforms". Nature Protocol 11 (2016): 727-746.

30. Noshadi I., et al. "In vitro and in vivo analysis of visible light crosslinkable gelatin methacryloyl (GelMA) hydrogels". Biomaterials 5 (2017): 2093-2105.

31. Meng K., et al. "A Reversibly Responsive Fluorochromic Hydrogel Based on Lanthanide-Mannose Complex". Advanced Sciences 6 (2019): 1802112.

32. Zhao X., et al. "Injectable antibacterial conductive nanocomposite cryogels with rapid shape recovery for noncompressible hemorrhage and wound healing". Nature Communication 9 (2018): 2784

33. Maulvi FA., et al. "A review on therapeutic contact lenses for ocular drug delivery". Drug Delivery 23 (2016): 3017-3026.

34. Maulvi FA., et al. "In vitro and in vivo evaluation of novel implantation technology in hydrogel contact lenses for controlled drug delivery. In vitro and in vivo evaluation of novel implantation technology in hydrogel contact lenses for controlled drug delivery". Journal of Controlled Release 226 (2016): 47-56.

35. Li YW and Lu HZ. "Dual Stable Nanomedicines Prepared by Cisplatin-Crosslinked Camptothecin Prodrug Micelles for E_ ective Drug Delivery". ACS Applied Materials and Interfaces 11 (2019): 20649-20659. 
36. Li J. "Self-assembled supramolecular hydrogels based on polymer-cyclodextrin inclusion complexes for drug delivery". NPG Asian Material 2 (2010): 112.

37. Moncada-Basualto M. "Supramolecular hydrogels of $\beta$-cyclodextrin linked to calcium homopoly-lguluronate for release of coumarins with trypanocidal activity". Carbohydrate Polymers 204 (2016): 170-181.

38. Jalalvandi E. "Cyclodextrin -polyhydrazine degradable gels for hydrophobic drug delivery". Materials Science and Engineering C 69 (2016): 144-153.

39. Thi TTH. "Oxidized cyclodextrin-functionalized injectable gelatin hydrogels as a new platform for tissue adhesive hydrophobic drug delivery". RSC Advances 7 (2017): 34053-34062.

40. Zhang L. "Multifunctional quantum dot DNA hydrogels". Nature Communication 8 (2017): 381.

41. Le X. "Fe3+-, pH-, Thermoresponsive Supramolecular Hydrogel with Multi shape Memory Effect". ACS Applied Materials and Interfaces 9 (2017): 9038-9044.

42. Cao W. " $\gamma$-Ray-Responsive Supramolecular Hydrogel Based on a Diselenide-Containing Polymer and a Peptide". Angewandte Chemie 125 (2013): 6353-6357.

43. Liang Y. "A cell-instructive hydrogel to regulate malignancy of 3D tumor spheroids with matrix rigidity". Biomaterials 32 (2011): 9308-9315.

44. Alvarez-Lorenzo C. "Bioinspired hydrogels for drug-eluting contact lenses”. Acta Biomaterial 84 (2019): 49-62.

45. Zhu Q. “Inner layer-embedded contact lenses for $\mathrm{pH}$-triggered controlled ocular drug delivery". European Journal of Pharmaceutics and Biopharmaceutics 128 (2018): 220-229.

46. Zhu Q. "Sustained ophthalmic delivery of highly soluble drug using pH-triggered inner layer-embedded contact lens". International Journal of Pharmaceutics 544 (2018): 100-111.

47. Segura T. "Materials with fungi-bioinspired surface for efficient binding and fungi-sensitive release of antifungal agents". Biomacromolecules 15 (2014): 1860-1870.

48. Kim H. "Synergistically enhanced selective intracellular uptake of anticancer drug carrier comprising folic acid-conjugated hydrogels containing magnetite nanoparticles". Scientific Report 7 (2017): 41090-41090.
49. Schlegel PN. "Effective long-term androgen suppression in men with prostate cancer using a hydrogel implant with the GnRH agonist histrelin". Urology 58 (2001): 578-582.

50. Wang X. "Vaginal delivery of carboplatin-loaded thermosensitive hydrogel to prevent local cervical cancer recurrence in mice". Drug Delivery 23 (2016): 3544-3551.

Volume 5 Issue 6 June 2021

(C) All rights are reserved by Shailee V Tiwari and Shruti V Dabhade. 